\title{
Conceptual Schematic Design of Evolving Bci Design Principles
}

\author{
A. Ramesh Babu, M. Jagadeeshwar, S. Suman Kumar
}

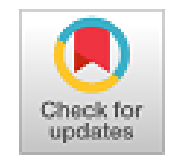

\begin{abstract}
At once of boosting disagreement and fast technological change, the ICRC requires each to understand the influence of brand new modern technologies on folks impacted through armed dispute and also to make altruistic answers that take care of the necessities of one of the most susceptible. Learning algorithms in lots of uses that's we take advantage of daily. Whenever a web online search engine like Google or Bing is made use of to browse the world wide web, some of the explanations that functions so properly is due to the fact that a learning formula, one applied by Google.com or even Microsoft, has actually learned exactly how to position website. Today, machine learning is a widespread condition that incorporates a lot of sorts of plans that you'll stumble upon in significant records analytics and records mining.
\end{abstract}

Index Terms : Machine Learning. AI, data mining

\section{INTRODUCTION}

At its the majority of essential amount, artificial intelligence refers to any sort of kind of pc plan that can easily "know" by itself without needing to be actually explicitly configured by a human. The expression (and also its actual idea) possesses its own sources decades back--completely to Alan Turing's critical 1950 paper "Processing Machinery and Intelligence," which included a section on his well-known "Discovering Maker" that may misdirect a private into experiencing that it is really actual.

Ultimately of the amount of time, the "thoughts" as a matter of fact powering really most anticipating training courses-featuring spam filters, item recommenders, as well as fraudulence sensors-- are artificial intelligence algorithms.

In administered learning, the customer trains the course to generate a solution based on a known and described records established. Category as well as regression formulas, consisting of random woods, choice trees, and also assistance vector machines, are actually frequently used for supervised learning duties.In without supervision artificial intelligence, the algorithms produce solutions on not known as well as unlabeled data. Information experts typically utilize without supervision procedures for discovering patterns in brand-new data collections.

Revised Manuscript Received on October 30, 2019.

* Correspondence Author

A. Ramesh Babu*, Associate Professor, Department of CS, Chaitanya Group of Colleges, India

M. Jagadeeshwar, Associate Professor, Department of CS, Chaitanya Group of Colleges, India

S. Suman Kumar, Associate Professor, Department of CSE, SVS Group of Institutions, India

(C) The Authors. Published by Blue Eyes Intelligence Engineering and Sciences Publication (BEIESP). This is an open access article under the CC BY-NC-ND license (http://creativecommons.org/licenses/by-nc$\underline{\mathrm{nd} / 4.0 /)}$
Clustering algorithms, like K-means, are actually usually used in unsupervised machine learning.Records researchers can easily set machine learning algorithms making use of a variety of innovations and also languages, including Espresso, Python, Scala, various other others.

They may also use pre-built machine learning platforms to accelerate the method; Mahout is an instance of a machine learning structure that was actually popular on Apache Hadoop, while Apache Fire's MLlib public library today has actually ended up being a requirement.

\section{DEEP LEARNING}

It is actually a type of artificial intelligence that may quickly capitalize on either dealt with or perhaps certainly not being actually enjoyed methods and even each. While it is really absolutely not always brand new, profound knowledge has actually simply lately viewed a rise in allure as a way to improve the solution of specific forms of hard pc troubles, most particularly in the personal computer outlook and also all-natural foreign language processing (NLP) areas. Semantic networks may have numerous covert coatings (all_is_magic/ Shutterstock) Deep learning is based on the embodiment learning (or even function learning) branch of artificial intelligence theory.

Through removing top-level, sophisticated abstractions as information embodiments by means of a hierarchical learning method, deep learning models yield results faster than conventional machine learning strategies, a deep learning type are going to definitely learn the functionalities that are very important on its own, as opposed to asking for the relevant information researcher to personally opt for the pertinent features, like the pointiness of ears situated in animal kitty pictures (considering that it in some way regularly returns to feline photographers in the future). The "deep" in deep learning derives from the different degrees that are really designed in too deep blue sea learning versions, which are actually typically neural networks. A convolutional semantic network (CNN) could be composed of numerous, several layers of designs, where each layer takes input from the previous layer, processes it, and also outputs it to the next layer, in a daisy-chain fashion trend. Deep learning is thus prominent today because of pair of main factors. Initially it was found that CNNs operate much a lot faster on GPUs, such as NVidia's Tesla K80 processor. The second thing is, records experts understood that the huge accumulations of information we have actually been actually collecting may work as an extensive instruction corpus and also consequently give a boost to the CNNs into giving considerable improvement in the accuracy of personal computer sight as well as NLP algorithms.

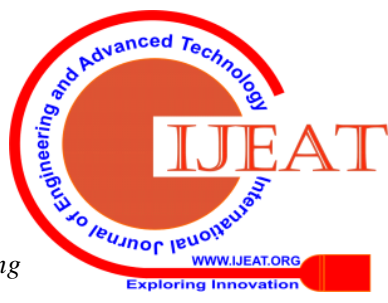


Tens or flow is actually an instance of a software progression platform, made through Google, that is finding a rise of passion; Caffe, Light, as well as Theano are other examples. Much of the progress in creating self-driving autos can be attributed to developments in deep learning using CNNs on GPUs, which possesses the mutual impact of helping to feed further breakthroughs in deep learning and also the broader expert system field.

\section{ADVANCES IN DATA INFRASTRUCTURE}

\section{New Sources}

Several new information resources have developed in the final years as essential ecosystems for exhibiting and also videotaping behavioral styles. Each information source has blind spots. On the one finger, a larger ecosystem could be anticipated to improve the chances of catching vital behavioral relevant information. Alternatively, a much smaller information ecosystem will demand much less modeling attempt and also might be even more expense efficient due to the fact that framework prices expand along with ecological community dimension, maybe faster than any benefits. Nevertheless, coming from a purely choices in viewpoint, one could think that additional information coming from more sources ought to-- various other points being actually equal-- boost ability for personality choices in. Some unique data streams have currently shown market value for behavioral choices in. For example, social media sites (SM) data has come to be common as well as is now an important analytical resource in national politics. Socialmedia platforms likewise function as tools for influencing discourse and for measuring behaviors/influence. Social networking site systems solution specific data as well as likewise relational or even network data. The ascendance of social networking sites platforms has caused a surge in procedures and also tools for dealing with relational/network/graph information. Cellular Information Records (CDRs), featuring metadata are additionally useful, particularly for deducing spatial behavioral tastes. Recent study indicates that CDRs are actually incredibly helpful for pinpointing spatial behaviors appropriate to, e.g., migration/disaster reaction buying styles and individual network affinities. The current- year developments related to "WikiLeaks" illustrate the real-life importance of even restricted metadata from cellular records reports.

Various other novel data streams consist of:

$>$ economic documents coming from financial institutions, retail records, and also financial innovation agencies (FinTech).

$>$ social and also personal online video security coming from road electronic cameras, and cell phone audios.

$>$ voice records: coming from Artificial Intelligence individual associates (Siri, Alexa, Google.com Now).

The growth in behaviorally appropriate information flows exemplifies an increasing reliance on tools and also units for mediating behaviors (e.g. DIRECTION FINDER for navigating, songs flows for state of mind management, social media platforms for phrase). It is actually currently usual to possess reports or signals of a person's programs, purposes, as well as mental states in electronic type, specifically in affluent lifestyles along with high mobile

phone adopting. Clark as well as utilized the condition "The Extended Thoughts" to pertain to the extension of psychological thought and also cognition outside the (as yet) unobservable boundaries of the individual thoughts. Lengthy thoughts with accessible digital information exhausts may be potentially advanced for behavioral choices in. The information environment seems to be developing because instructions.

\section{LEGAL BASIS FOR HUMAN CONTROL IN ARMED CONFLICT}

For battle people, individual control over AI and also machine-learning applications utilized as ways and approaches of war is actually demanded to guarantee observance with the rule. The rules of global altruistic legislation are actually addressed to people. It is actually people that comply with and implement the legislation, and also it is actually human beings who will certainly be held accountable for violations. In particular, fighters possess a special commitment to help make the common sens called for of all of them due to the global humanitarian regulation guidelines controlling the conduct of hostilities, as well as this responsibility can easily certainly not be actually transferred to a machine, an item of software or even a formula. These guidelines need context-specific judgements to become taken through those who prepare, choose and accomplish attacks to guarantee: difference-- between army objectives, which may legally be assaulted, as well as civilians or even private objects, which have to certainly not be struck; symmetry-- in regards to making sure that the secondary noncombatant danger gotten out of an assault is going to certainly not be excessive in connection with the concrete and also direct army advantage prepared for; as well as to permit measures in assault-- to ensure risks to private citizens may be further minimized. Where AI systems are used in attacks-- whether as portion of bodily or even cyber-weapon units, or even in decision-support systems-- their concept as well as usage need to make it possible for fighters to produce these judgements. With respect to autonomous tool devices, the States event to the Convention on Certain Regular Weapons (CCW), have identified that "human accountability" for the use of tool bodies and also the use of power "must be actually retained", and also numerous States, worldwide organizations-- including the ICRC-- and also civil culture companies, have actually emphasized the requirement for individual management to make certain compliance along with worldwide humanitarian law and also being compatible along with reliable market values.

Beyond making use of force and also targeting, the prospective use of AI units for other selections overseen by certain rules of international altruistic legislation are going to likely need careful factor of required human command, as well as common sense, such as in apprehension.

\section{ETHICAL BASIS FOR HUMAN CONTROL}

Arising uses of Artificial Intelligence as well as machine learning have additionally brought honest questions to the cutting edge of social dispute. 
A typical aspect of overall "Artificial Intelligence Principles" created and acknowledged through federal governments, researchers, ethicists, study principle and also technology firms is the relevance of the human factor to make certain lawful conformity and also honest acceptability. As an example, the 2017 Asilomar Artificial Intelligence Principles stress placement with human worths, compatibility along with "individual dignity, civil liberties, flexibilities and also cultural diversity", and human management; "humans ought to opt for just how and also whether to hand over selections to AI units, to complete human-chosen goals". The European Commission's HighLevel Pro Team on Expert system stressed the relevance of "human company as well as administration", such that AI systems ought to "sustain human autonomy and decisionmaking", as well as ensure individual administration by means of human-in-the-loop, human- on-the-loop, or humanin-command techniques. The organization for Economic Cooperation as well as Development (OECD) Principles on Expert system-- used in Might 2019 through all 36 participant States, together with Argentina, Brazil, Colombia, Costa Rica, Peru and Romania-- highlight the significance of "human-centred worths as well as fairness", indicating that customers of Artificial Intelligence "ought to carry out systems and also guards, such as capability for human judgment, that are appropriate to the context and regular along with the state of fine art". The Beijing Artificial Intelligence Guidelines, embraced in May 2019 by a team of leading Chinese analysis principle and also modern technology companies, condition that "continual initiatives must be actually created to boost the maturity, toughness, reliability, as well as controllability of AI systems" and also urge "explorations on Human-AI control ... that would certainly provide full play to human benefits and also features". A lot of private technology firms have actually also released Artificial Intelligence Principles highlighting the relevance of individual command, particularly for vulnerable requests presenting the threat of danger, and also highlighting that the "function of $\mathrm{AI}$... is to enhance-- not substitute-- human intelligence".

A similar request of $\mathrm{AI}$ and artificial intelligence in the electronic sphere, is the use of these resources for information combat, especially the creation and dispersing of incorrect relevant information with intent to scam-- i.e. disinformation-- along with the dispersing of false info without such intent-- i.e. misinformation. Certainly not all include AI and artificial intelligence, but these technologies seem set to alter the attributes and also scale of the control of info in combat and also the potential outcomes. AIenabled bodies have been commonly used to create fake information-- whether content, audio, pictures or even online video-- which is progressively complicated to distinguish from genuine details. Use these devices through disagreement celebrations to boost olden approaches of brainwashing to maneuver opinion and influence choices might possess significant implications on the ground. For the ICRC, there are problems that civilians might, due to electronic disinformation or false information, undergo arrest or even ill-treatment, discrimination or even rejection of accessibility to necessary companies, or attacks on their individual or even residential property.

\section{ARTIFICIAL INTELLIGENCE AND MACHINE LEARNING}

Expert system and ML are quite associated. According to McCarthy, some of the owners of the area,

AI is actually "the science and also engineering of creating intelligent machines, specifically smart computer plans. It belongs to the identical duty of using pcs to know individual intelligence, but AI does not must limit itself to procedures that are actually biologically observable."

This is actually reasonably common and consists of multiple tasks including abstractly thinking as well as generalizing regarding the globe, handling puzzles, considering how to attain goals, relocating about in the globe, identifying items and noises, speaking, equating, carrying out social or even organisation transactions, artistic work (e.g., developing fine art or even poetry), and also handling robots. Additionally, the habits of a machine is actually certainly not only the end result of the course, it is additionally impacted by its "body" as well as the enviroment it is literally installed in. To maintain it simple, having said that, if you can create an extremely clever course that possesses, state, human-like behavior, it could be AI. However unless it automatically profits from data.

So, AI as well as ML are each regarding building intelligent pc courses, as well as DL, being an occasion of ML, is actually no exception. Deep, which has actually accomplished amazing increases in several domain names spanning coming from object acknowledgment, pep talk recognition, as well as management, may be considered as creating computer plans, specifically setting levels of absorption in a differentiable method making use of reusable structures including convolution, merging, car encoders, variational reasoning networks, and so on. Simply put, our company replace the intricacy of writing algorithms, that cover every scenario, along with the intricacy of locating the right standard summary of the algorithms-- in the form of, for instance, a deep semantic network-- and refining records. By virtue of the generalization of neural networks-- they are overall function approximators-- educating all of them is data hungry and normally calls for huge labeled instruction sets. While benchmark instruction collections for object acknowledgment, establishment hundreds or even hundreds of examples per class label, for lots of AI applications, producing tagged instruction information is actually the absolute most time-consuming and expensive part of DL. Learning to play video games might need numerous hours of instruction adventure and/or really expensive computing energy. On the other hand, creating an AI protocol that covers every event of a task to address, point out, thinking about data as well as understanding to label data and, in turn, create, for example, DL much less data-hungry-- is actually a great deal of manual labor, however we understand what the protocol carries out by design and that it may study which it can easily extra effortlessly know the complication of the issue it fixes. When a machine has to engage along with a human, this seems to be to become specifically valuable. 
This emphasizes that ML and also AI are actually definitely similar, but not quite the exact same. Expert system concerns complication dealing with, thinking, and also learning typically. Artificial intelligence is exclusively about learning-- picking up from instances, from definitions, from being told, as well as from habits. The easiest means to think of their connection is to picture all of them as concentric circles along with AI first and ML sitting inside (with DL proper inside each), given that ML additionally calls for composing algorithms that cover every event, specifically, of the learning procedure. The critical point is actually that they share the suggestion of using estimation as the foreign language for intelligent actions. What kind of computation is actually utilized as well as just how should it be actually programed? This is actually not the ideal inquiry. Calculation not either eliminate hunt, sensible, probabilistic, and restraint shows procedures nor (deep) (un) closely watched and reinforcement learning methods, to name a few, but does, as a computational design, include all of these procedures.

Rethinking AlphaGo: AlphaGo and its own successor AlphaGo Absolutely no both blend DL and also tree hunt-ML and AI. Additionally, the "Allen Artificial Intelligence Scientific Research Challenge" ought to be thought about. The duty was actually to comprehend a paragraph that states a science problem, at the secondary school degree and after that to address a multiple-choice inquiry. All winning versions used ML however, stopped working to pass the examination at the level of a competent mid schooler. All victors debated that it was crystal clear that applying a much deeper, semantic level of reasoning with scientific knowledge to the inquiry and answers, is the vital to attaining true intelligence. In other words, Artificial Intelligence has to deal with knowledge, thinking, as well as learning, using configured and learning-based set designs in a bundled style.

\section{RESULTING SIGNAL PROCESSING FOR BRAIN- COMPUTER INTERFACES (BCI)}

A BCI or even Human Brain Pc Interface is actually cooperation between a mind and a tool or machine that reads the electrical indicators coming from the human brain the uses them to guide some exterior activities like moving the cursor or even a prosthetic branch. The unit or machine serves as a user interface in between the brain as well as the contest be managed by the brain. Figure 1 presents a theoretical schematic overview of progressing BCI layout concepts. Records gotten coming from sensing units and devices within, on, and around an individual subject matter are actually changed into insightful representations by means of domain-specific sign pre-processing. The leading signs are integrated to create psychomotor state representations. These quotes may be offered to the devices the target is actually engaging with.

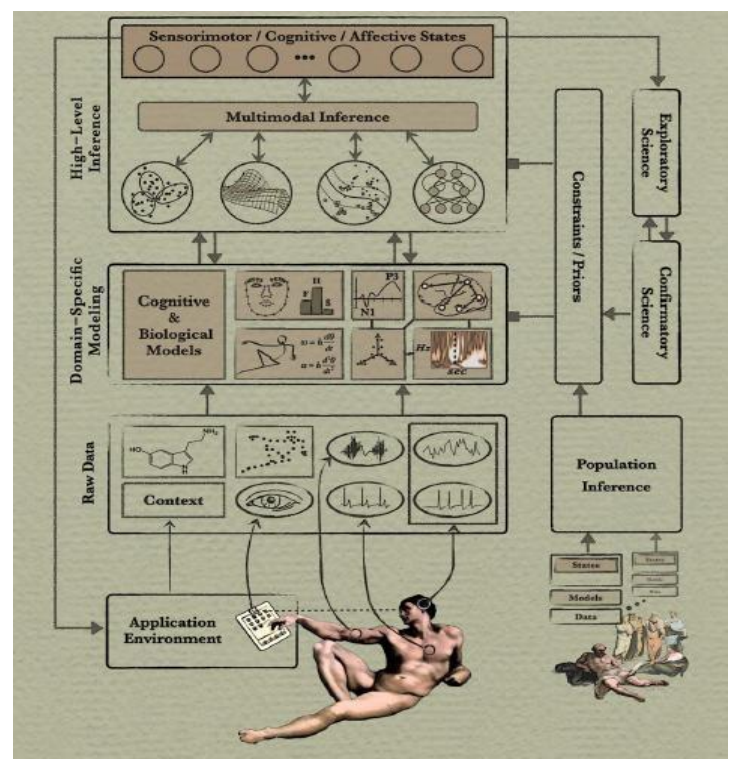

Figure 1: Conceptual schematic overview of evolving BCI design principles

\section{CONCLUSION}

Numerous fields and also investigation locations need to have to collaborate to drive these advancements. Making use of estimation as the typical language possesses the possibility for progressing learning principles as well as presuming relevant information that is actually each easy and also difficult for humans to acquire.

To this end, the "Artificial Intelligence and Artificial intelligence" in Frontiers in Big Information accepts fundamental and used documents and also duplication research studies from a large range of subjects founding ML, Artificial Intelligence, as well as their exchange. It will certainly cultivate the scholarly conversation of the causes and effects of accomplishments providing a correct standpoint on the obtained outcomes.

\section{REFERENCES}

1. Tzanis, George, et al. "Modern Functions of Machine Learning." Process of the first Annual SEERC Doctoral Student Event-- DSC. 2006.

2. Horvitz, Eric. "Artificial intelligence, presuming, and also knowledge in each day lifestyle: Guidelines as well as additionally complications." Process of. Vol. 360. 2006.

3. Mitchell, Tom Michael. The industry of expert system. Carnegie Mellon Educational Institution, College of Infotech, Machine Learning Group, 2006.

4. Wiese, Bénard, and also Religious Omlin. Charge card deals, fraud discovery, and machine learning: Modelling time with LSTM frequent neural networks. Springer Berlin Heidelberg, 2009.

5. Kumar, Vinod, as well as Dr Om Prakash Sangwan. "Signature Based Invasion Detection System Using SNORT." International Diary of Pc Treatments \& Information Technology 1 (2012 ).

6. Shen, Shunrong, Haomiao Jiang, and also Tongda Zhang. "Stock exchange projecting making use of artificial intelligence protocols." (2012).

7. Pain, Bo, Lillian Lee, and Shivakumar Vaithyanathan. "Thumbs up?: conviction distinction making use of machine learning methods." Process of the ACL-02 conference on Empirical approaches in organic language processing-Volume 10. Organization for Computational Grammar, 2002. 